\title{
Kongreßkalender 2001
}

Für die Aufnahme neuer Veranstaltungen in den Kongreßkalender senden Sie bitte die entsprechenden Informationen an:

\author{
Dr. Francesco Dati \\ TÜV. Rheinland Product Safety GmbH \\ Am Grauen Stein \\ D-51105 Köln/Germany \\ Tel.: 0221-8061685 \\ Fax: 0221-8061358 \\ E-Mail: dati@de.tuv.com
}

Für eine ausführliche Liste wissenschaftlicher Veranstaltungen siehe LaboratoriumsMedizin, Heft 8/2000 (Seite 384-392).

22. - 23. Januar 2001

Frankfurt

11. - 12. Februar 2001 Newport Beach, CA, USA

14. - 17. Februar 2001 Düsseldorf

8. - 11. März 2001

Dubronovik, Kroatien

8. - 11. März 2001

Genf. Schweiz

15. - 18. März 2001

Wien, Österreich

18. - 21. März 2001

Orlando, FL, USA

1. - 4. April 2001

Instanbul, Turkey

19. - 21. April 2001

Mannheim

30. April - 4. Mai 2001

London, UK
Statusseminar: „Chiptechnologien: Vom Genom zum Proteom“

Sekr.: DECHEMA e.V.; Frau Xueqing Wu /AG Tagungen, Theodor-Heuss-Allee 25. D-60486 Frankfurt,

Tel.: 0697564 152; Fax: 0697564 176, E-Mail: wu@dechema.de,

Internet: www.dechema.de/deutsch/veransta/chips.01/pages/i_chips $1 . h t m$

24 $4^{\text {th }}$ Annual Arnorld O. Beckman Conference - The Clinician and Laboratorian: Dynamic Transitions

Sekr: AACC Customer Service, 2101 L Street, NW, Suite 202, Washington, DC 20037 - 1526. USA,

Tel.: (1) 202 857 0717; Fax: (1) 202833 4576. E-mail: custserv@aacc.org,

Internet: http://www.aacc.org/meetings/oakridge/

45. Jahrestagung der Gesellschaft für Thrombose- und Hämostaseforschung (GTH) Sekr.: Congress Partner GmbH. Birkenstr. 37, D-28195 Bremen, Tel.: 0421 303131; Fax: 0421 303133, E-mail: bremen@cpb.de,.Internet: www.cpb.de/gth2001.html

IFCC General Conference

Sekr.: IFCC Office, Centre du Médicament,30 rue Lionnois, F-54000 Nancy, Frankreich,

Tel.: (33) 3833526 16; Fax: (33) 3833213 22, E-mail: chantal.thirion @ifcc.u-nancy.fr.

Internet: http://www.ifcc.org

European Atherosclerosis Society: Workshop on the Immune System in Atherosclerosis

Sekr.: EAS c/o KENES, P.O. Box 50006, Tel Aviv 61500, Israel, Tel.: (972) 35140 018;

Fax: 972) 35140 077, E-mail: immune@kenes.com, Internet: www.kenes.com/immune-eas

5. Symposium der Interdisziplinären Gruppe für Labor und Durchflusszytometrie (IGLD)

Sekr.: Dr. Gerhard Fritsch, CCRI, St. Anna Kinderspital, Kinderspitalgasse 6. A-1090 Wien, Österreich:

Fax: (43) 1408 7230, E-mail: zavadil@ccri.univie.ac.at, Internet: www.igld.de

American College of Cardiology - $50^{\text {th }}$ Annual Scientific Session

Sekr.: American College of Cardiology. 9111 Old Georgetown Road, Bethesda. Maryland 20814-1699, USA, Tel.: (1) 301897 5400; Fax: (1) 301897 9745, E-mail: annualmeeting@acc.org,

Internet: www.acc.org/meetings

$11^{\text {th }}$ Congress of Clinical Microbiology and Infectious Diseases

Sekr.: ESCMID Executive Office, c/o AKM Congress Service, P.O. Box, CH-4005 Basel/Switzerland, Tel.: (41) 616867711 ; Fax: (41) 61686 7788, E-mail: info@akm.ch, Internet: http://www.akm.ch

67. Jahrestagung der Deutschen Gesellschaft für Kardiologie - Herz- und Kreislaufforschung (DGK)

Sekr.: DGK-Geschäftsstelle, Goethestr. 38, 40237 Düsseldorf. Tel.: 02116006 920; Fax: 021160069210.

E-mail: dgk@uni-duesseldorf.de, Internet: http://www.dgkardiol.de

Focus 2001. National Meeting of the Association of Clinical Biochemists

Sekr.: Focus 2001, PO Box 409. Cambridge CB1 4QD, UK, Tel.: (44) 1223 51611)3; Fax: (44) 1223

500978, E-mail: info@focus-acb.org, Internet: http://www.focus-acb.org 
4. - 5. Mai 2001

Scattlc. WA, USA

20. - 23. Mai 2001

Glasgow. UK

25. - 26. Mai 2001

Prag, Tschechische Republik

26. - 31. Mai 2001

Prag, Tschechische Republik

14. - 16. Juni 2001 Rom. Italien

15. - 16. Juni 2001 Hamburg

24. - 27. Juni 2001 Wien, Österreich

6. - 12. Juli 2001

Paris, Frankreich

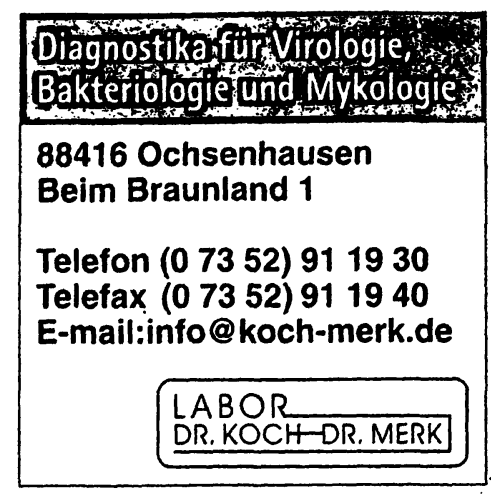

$33^{\text {rd }}$ Annual Oak Ridge Conference - „The Post Genome Era - New Targets and New Technologies"

Sekr.: AACC Customer Service, 2101 L Street. NW, Suite 202, Washington, DC 20037 - 1526. USA,

Tel.: (1) 2028570717 ; Fax: (1) 202833 4576, E-mail: custserv@aacc.org.

Internet: http://www.aacc.org/meetings/oakridge/

$7^{\text {nd }}$ Meeting of the European Atherosclerosis Society

Sekr.: Prof. Jim Shepherd, Dept. Pathological Biochemistry, Glasgow Royal Infirmary, Glasgow G4 OSF, Scotland, UK. Tel.: (44) 141552 0689; Fax: (44) 1415531703

\section{IFCC/Beckman Coulter European Conference}

Medical Applications of Protein Research

Sekr.: Protein Conference Secretariat, Beckman Coulter Eurocenter SA, P.O. Box 301, CH-1260 Nyon, Schiveiz, Tel.: (41) 22994 3437; Fax: (41) 22994 3467, E-mail: proteins@beckmancoulter.com

EUROMEDLAB $-14^{\text {th }}$ European Congress of Clinical Chemistry and Laboratory Medicine (IFCC Congress) 2001 PRAHA

Sekr.: EUROMEDLAB Secretariat, Institute for Clinical Biochemistry \& Diagnostics; University Hospital, CZ-50005 Hradec Kralove, Tschechische Republik, Tel.: (420) 49583 3040; Fax: (420) 495832003 ,

E-mail: euromedlab@lfhk.cuni.cz, Internet: http://www.congress.cls.cz/euromedlab2001/

\section{Course: The Role of „Chest Pain Unit“" in Risk Stratification and Management of} Patients with Chest Pain

Sekr.: The European Heart House (ECOR), ETP \& in-House Meetings Derepartment, 2035 Route des Colles - Les Templiers, P.O. Box 179, F-06903 Sophia Antipolis Cedex. Frankreich, Tel.: (33) 492947600 ; Fax: (33) 49294 7601, E-mail: webmaster@escardio.org, Internet: http://www.escardio.org/

44. Hamburger Symposion über Blutgerinnung und klinische Hämostaseologie Sekr:: Prof. Dr. F. Reinhard Mathias, Zentrum für Innere Medizin des Klinikums der Universität, Klinikstr. 36, D-35385 Giessen, Tel.: 0641994 2131; Fax: 0641994 2139,

E-mail: reinhard.f.matthias@innere.med.uni-giessen.de, Internet: http://www.pharma.roche.de/hsbg

$38^{\text {th }}$ Congress of the European Renal Association and the European Dialysis and Transplant Association

Sekr.: ERA-EDTA Congress Office, Casella Postale 474 Parma Sud, l-43 100 Parma, Italien,

Tel.: (39) 0521 989078; Fax: (39) 0521 291777, E-mail: eraedta@ipsuniv.cc.unipr.it.

Internet: http://www.unipr.it-eraedta

$18^{\text {th }}$ Congress of the International Society on Thrombosis and Haemostasis $47^{\text {th }}$ Meeting of the Scientific \& Standardisation Committee

Sekr.: Convergences - ISTH 2001, 120, rue de la Croix-Faubin, F-75557 Paris Cedex 11, Frankreich, Tel.: (33) 14464 1515; Fax: (33) 14464 1516, E-mail: isth2001@ @onvergences.fr,

Internet: http://www.isth2001.com 